\title{
Caught in the Act: In Vivo Mapping of Macrophage Infiltration in Nerve Injury by Magnetic Resonance Imaging
}

\author{
Martin Bendszus ${ }^{1 \star}$ and Guido Stoll ${ }^{2 *}$ \\ Departments of ${ }^{1}$ Neuroradiology and ${ }^{2}$ Neurology, University of Würzburg, D-97080 Würzburg, Germany
}

\begin{abstract}
In vivo tracking of hematogenous macrophages has been a major challenge because these cells are key players in nerve injury and repair. We visualized the spatiotemporal course of macrophage infiltration after acute peripheral nerve injury in living rats by using superparamagnetic iron oxide (SPIO) particles and magnetic resonance imaging (MRI). A signal loss on MR images indicating iron accumulation was present in degenerating sciatic nerves between days 1 and 8 after a crush lesion, ceased thereafter, and corresponded to the transient presence of iron-labeled ED1-positive macrophages in tissue sections. In contrast, no SPIO accumulation was seen after optic nerve crush, which revealed microglial activation but lacked macrophage infiltration. SPIO-enhanced MRI provides a new tool to selectively visualize active periods of macrophage transmigration into the nervous system, thus enabling dynamic views on a fundamental process in a multitude of nerve disorders.
\end{abstract}

Key words: nerve injury; Wallerian degeneration; macrophage; MRI; SPIO

\section{Introduction}

Macrophages play a pivotal role in the pathophysiology of numerous neurological disorders (Benveniste, 1997; Stoll and Jander, 1999; Kiefer et al., 2001; Williams and Hickey, 2002). In the CNS, infiltration by hematogenous macrophages often overlaps with activation of resident microglia. Because of phagocytic transformation and common phenotypical activation markers, the two cell populations thereby become indistinguishable in tissue sections (Flaris et al., 1993; Stoll and Jander, 1999). Magnetic resonance imaging (MRI) has emerged as an indispensable tool for assessing structural changes in the nervous system. Changes in MR signals, however, reflect gross tissue alterations but do not depict histological details such as accumulation of specific cell types (Higer and Bielke, 1990). So far only positron emission tomography using the ligand PK11195 delineated further cellular details, but macrophage and microglial responses could not be differentiated (Banati, 2002). In the present study, we report on the in vivo monitoring of macrophage infiltration in nerve lesions by use of superparamagnetic iron oxide (SPIO) particles and MRI and their differentiation from microglial responses.

Crush of nerves or fiber tracts is an injury model that leads to degeneration of the distal stump, a process designated Wallerian degeneration (WD) (Waller, 1850). On MRI, WD leads to a uniform and persistent hyperintensity on T2-weighted (T2-w) images that does not change during the evolving inflammatory re-

Received April 30, 2003; revised July 25, 2003; accepted Aug. 21, 2003.

This work was supported by funds from the state of Bavaria. We thank C. Wesemeier, T. Horn, and K. Treutlein for expert technical assistance, Prof. M. Koltzenburg (Institute of Neurology, Queen Square, London) for contribution to pilot experiments, and Profs. L. Solymosi and K. V. Toyka (University of Würzburg) for support and critical reading of this manuscript.

*M.B. and G.S. equally contributed to this work.

Correspondence should be addressed to Dr. M. Bendszus, Department of Neuroradiology, University ofWürzburg Josef-Schneider-Strasse 11, D-97080 Würzburg, Germany. E-mail: bendszus@neuroradiologie.uni-wuerzburg.de. Copyright $\odot 2003$ Society for Neuroscience $\quad$ 0270-6474/03/2310892-05\$15.00/0 sponse and resolves after successful regeneration (Kuhn et al.; 1988, Titelbaum et al., 1989; Dailey et al., 1997; Grant et al., 2002). During WD a cellular environment is created in the peripheral nervous system (PNS) that facilitates prompt nerve regeneration (Ramon y Cajal, 1991; Stoll and Müller, 1999; Snider et al., 2002). It is assumed that the rapidly evolving macrophage infiltration plays a crucial role in this process (Perry et al., 1987; Stoll et al., 1989a; Brück, 1997; David and Ousman, 2002). In contrast, macrophages are virtually excluded from entry after fiber tract or optic nerve $(\mathrm{ON})$ crush lesions in the CNS, where no spontaneous regeneration occurs (Ramon y Cajal, 1991; Horner and Gage, 2000; David and Ousman, 2002). Instead, resident microglia become activated but only slowly undergo phagocytic transformation (Perry et al., 1987; Stoll et al., 1989b; George and Griffin, 1994). We used WD of sciatic and optic nerves as lesion paradigms, allowing a distinction between rapidly evolving macrophage infiltration in the PNS and pure microglial activation in the CNS to evaluate SPIO-enhanced MRI as a new tool to monitor macrophage migration into the nervous system in vivo.

\section{Materials and Methods}

Animal models. Thirty-six male Sprague Dawley rats (250-300 gm) were anesthetized with intraperitoneal injections of $100 \mathrm{mg} / \mathrm{kg}$ ketamin (Ketanest) and $10 \mathrm{mg} / \mathrm{kg}$ xylazin (Rompun). In one group $(n=29)$ the left sciatic nerve was exposed at mid-thigh level and crushed for $60 \mathrm{sec}$ using a small forceps; three additional animals were sham-operated. In a second set of experiments the left sciatic nerve was crushed in four rats, and a second crush was performed $6 \mathrm{~d}$ later on the contralateral nerve. An inhalation narcosis with enflurane in a $2: 1 \mathrm{~N}_{2} \mathrm{O} / \mathrm{O}_{2}$ atmosphere via a face mask was performed in 27 male Wistar rats (250-300 gm), and the optic nerve was crushed for $60 \mathrm{sec}$ immediately behind the eyeball. Animal studies were approved by the State of Bavaria.

As a contrast medium, commercially available superparamagnetic iron oxide particles (Resovist; Schering, Berlin, Germany) were applied at a dose of $0.2 \mathrm{mmol} F$ per kilogram of body weight by intracardial 

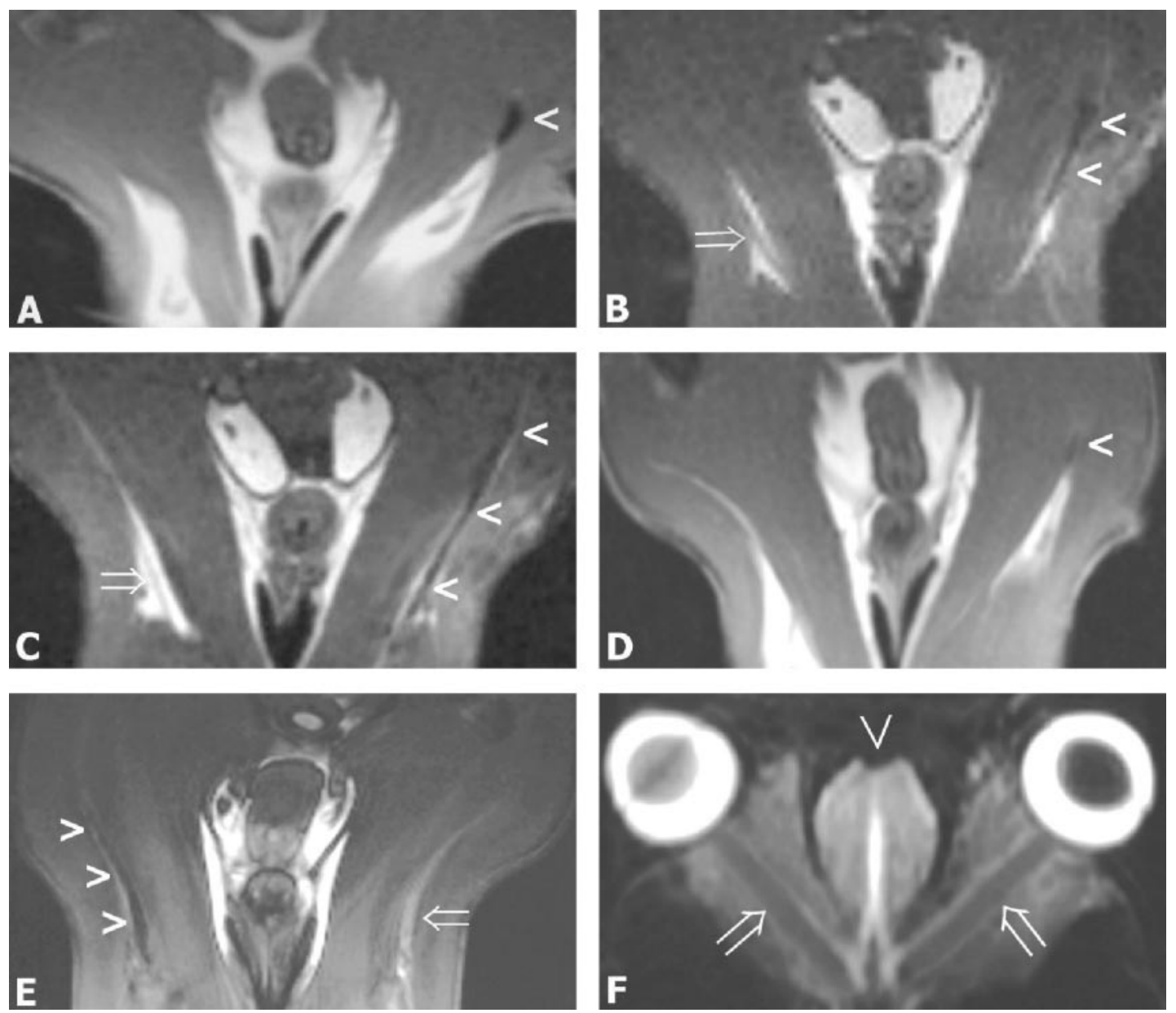

Figure 1. MRI of crushed and normal sciatic $(A-E)$ and optic nerves $(F)$ : coronal MR images (CISS sequence; slice thickness, $1 \mathrm{~mm})$ at days $1(A), 3(B), 5(C)$, and $8(D)$ after a proximal crush lesion of the left sciatic nerve. SPIO particles were given intracardially $24 \mathrm{hr}$ before MRI at each time point. On day 1, a focal signal loss (arrowhead) is present at the proximal sciatic nerve corresponding to the lesion site $(A)$. On days 3 and 5 , the signal loss extends farther into distal nerve segments ( $B, C$, arrowheads). Arrows point to the contralateral normal sciatic nerve revealing an isointense signal to the surrounding soft tissue. On day 8 , there is only a moderate signal loss at the lesion site ( $D$, arrowhead). E shows a coronal MR image at day 11 after crush of the left sciatic nerve and at day 5 after crush of the right sciatic nerve in the same animal (sequential crush). Iron-induced signal loss is restricted to the recently crushed right sciatic nerve (arrowheads), whereas the left sciatic nerve crushed $11 \mathrm{~d}$ before in the more chronic stage of Wallerian degeneration shows a signal increase (arrow). F, Semicoronal MR image (CISS sequence; angulation along the axis of both optic nerves; slice thickness, $1 \mathrm{~mm}$ ) $4 \mathrm{~d}$ after retro-orbital crush lesion of the left optic nerve and $24 \mathrm{hr}$ after SPI0 application. Arrowhead denotes olfactory bulb; arrows point to optic nerves. In contrast to the sciatic nerves, no focal signal loss is present in the crushed optic nerve.

injection usually $24 \mathrm{hr}$ before MRI and tissue removal for histological analysis $(n=47)$. In additional experiments, nine rats were crushed at the sciatic nerve, received SPIO particles immediately after crush, and were scanned by MRI with a delay of 1,3 , or $5 \mathrm{~d}$.

Magnetic resonance imaging. MR measurements were performed on a clinical 1.5 T unit (Magnetom Vision; Siemens, Erlangen, Germany). For anesthesia the same intraperitoneal protocol was used as for surgery. Animals with a sciatic nerve crush lesion were lying in prone position with both legs positioned in a round surface coil (diameter, $4 \mathrm{~cm}$ ). Animals with an optic nerve crush lesion were lying in supine position with the head fixed in a round surface coil (diameter, $4 \mathrm{~cm}$ ). The MR protocol included T1-w [repetition time (TR), $460 \mathrm{msec}$; echo time (TE), $14 \mathrm{msec}$ ] and T2-w (TR, $2500 \mathrm{msec}$; TE, $80 \mathrm{msec}$ ) sequences in the axial plane with a slice thickness of $3 \mathrm{~mm}$. Moreover, a three-dimensional constructed interference in steady state (CISS) sequence (TR, $16.4 \mathrm{msec}$; TE, 8.2 msec) (Casselman et al., 1993) was applied in the axial and coronal plane with a slice thickness of $1 \mathrm{~mm}$. MRI was performed in sham-operated rats and rats with sciatic nerve crush at days $1,3,5,8$, and 14. At each time point, three to four animals were examined. Rats with the sequential crush lesions were examined on day 11 after the first crush, which corresponds to day 5 of the second crush. Animals with optic nerve lesions were scanned on days 4 and 14 .

Histological and immunocytochemical studies. After MRI, rats were killed by an overdose of $\mathrm{CO}_{2}$; sciatic nerves and the intracranial proportions of crushed optic nerves were quickly removed, fixed by immersion in $4 \%$ paraformaldehyde, and embedded in paraffin. Additional optic nerves were collected at 1, 2, 7, 21 and $28 \mathrm{~d}$ after crush for histological analysis only. Paraffin sections (10 $\mu \mathrm{m}$ thick) were cut, deparaffinized with xylene, rehydrated, and washed in water and PBS. For iron staining, tissue sections were rinsed in deionized water and immersed in Perl's solution containing $2 \%$ potassium ferrocyanide and $2 \% \mathrm{HCl}$ at a $1: 1$ concentration for $30 \mathrm{~min}$. Sections were then rinsed in deionized water, dehydrated, and coverslipped, or further processed for ED1 immunocytochemistry. Additional sections were stained by immunocytochemistry using the antibody ED1, a marker for monocytes and macrophages (Serotec, Oxford, UK), at a dilution of 1:1000. Binding of ED1 antibodies to macrophages was visualized by the avidin-biotin-peroxidase method with diaminobenzidine as chromogen according to routine procedures (Stoll et al., 1989a). To assess the cellular distribution of iron particles, in addition we combined Perl's stain with ED1 immunocytochemistry. Sections were examined with a Zeiss-Axiophot microscope.

\section{Results}

In normal sciatic nerves, the nerve signal on MRI was isointense to the surrounding soft tissue and did not change after systemic 
application of SPIO particles. Similarly, exposing sciatic nerves without crush by sham operation did not change the MR signal. Without injection of SPIO particles, the distal segment of crushed sciatic nerves became hyperintense on T2-w images as reported previously (Kuhn et al., 1988; Titelbaum et al., 1989; Dailey et al., 1997; Grant et al., 2002). After application of SPIO particles, we observed a signal loss of the crushed nerves on MR images that developed with a proximodistal gradient and was indicative of local iron accumulation (Fig. 1). The signal loss was best seen on CISS images. SPIO particles were always injected intracardially $24 \mathrm{hr}$ before MRI. At day 1, a marked hypointensity was present at the lesion site on MR images on the crushed left but not the unaffected right sciatic nerves, which served as controls (Fig. 1A). Signal loss further extended distally in animals scanned at days $3(\sim 8 \mathrm{~mm})$ and $5(\sim 12 \mathrm{~mm})$ that had received SPIO particles on days 2 or 4 , respectively (Fig. 1B,C). Surprisingly, $8 \mathrm{~d}$ after nerve injury, there was only a focal signal loss at the lesion site, whereas more distal nerve segments were spared in contrast to days 3 and 5 (Fig. 1D). Rats examined $14 \mathrm{~d}$ after crush did not exhibit any signal loss in nerve segments undergoing WD. These MR findings indicated that iron contrast-mediated signal loss in degenerating nerves was a transient event.

After MRI, sciatic nerves were removed and fixed, and paraffin sections were immersed in Perl's solution to detect iron deposition and stained by immunocytochemistry using the antibody ED1 to document macrophage infiltration. In accordance with our MRI findings (Fig. 1A), we found focal accumulation of iron-positive cells at the crush site on day 1 (Fig. 2 A). Iron-positive cells were mainly associated with vessel walls; some appeared to transmigrate into the endoneurium (Fig. 2 B). At days 3 and 5, the number of iron-positive blue cells had increased concomitantly with the number of ED1-positive macrophages, and their distribution further extended into more distal parts of the degenerating nerves (Fig. $3 A, C$ ), paralleling our MRI findings (Fig. $1 B, C$ ). Ironpositive cells now were often associated with degenerating nerve fibers and located close to ovoids of degenerating myelin, which is typical for macrophages (Fig. 2C) (Stoll et al., 1989a). At day 8, only small foci of iron-positive cells were still present in degenerating nerves (Fig. $3 E$ ). On day 14, virtually no iron-positive cells were found within the endoneurium (Fig. 3G), despite the persistence of high numbers of ED1-positive macrophages (Fig. $3 \mathrm{H}$ ), which reside in injured nerves for months (Kuhlmann et al., 2001). Thus, passive diffusion of SPIO through the defective blood-nerve barrier and local uptake is unlikely because then one would expect continuing iron uptake in the presence of macrophages. Rather, the intracardially applied SPIO particles must have been phagocytosed by monocytes and macrophages in the circulation and were actively transported into the nerves by cel- lular migration. Accordingly, by immunocytochemistry on sections first immersed in Perl's solution, we could show that endoneurial monocytes with small dots of ED1 immunoreactivity, as well as postphagocytic macrophages within degenerating nerve fibers, revealed extensive colocalization of iron and ED1 (Fig. $2 D, E)$.

We next examined whether iron-labeled macrophages that have infiltrated the crush site further migrate intraneurally to the distal stump. Nine rats received SPIO at the time point of sciatic nerve crush, and groups of three each were examined by MRI at days 1,3 , and 5 . Signal loss remained restricted to the crush site in all animals regardless of the date of MRI at days 1, 3, or 5. This indicates that hematogenous macrophages remain stationary after nerve infiltration.

To further prove that iron accumulation is a transient event reflecting periods of monocyte and macrophage entry into crushed nerves, we performed a sequential crush experiment. In four animals, SPIO particles were injected $10 \mathrm{~d}$ after crushing the left sciatic nerve and $4 \mathrm{~d}$ after crushing the right sciatic nerve. MRI $24 \mathrm{hr}$ later revealed iron-induced signal loss in the 5-d-old right nerve crush, whereas the left 11-d-old crush did not exhibit signal 

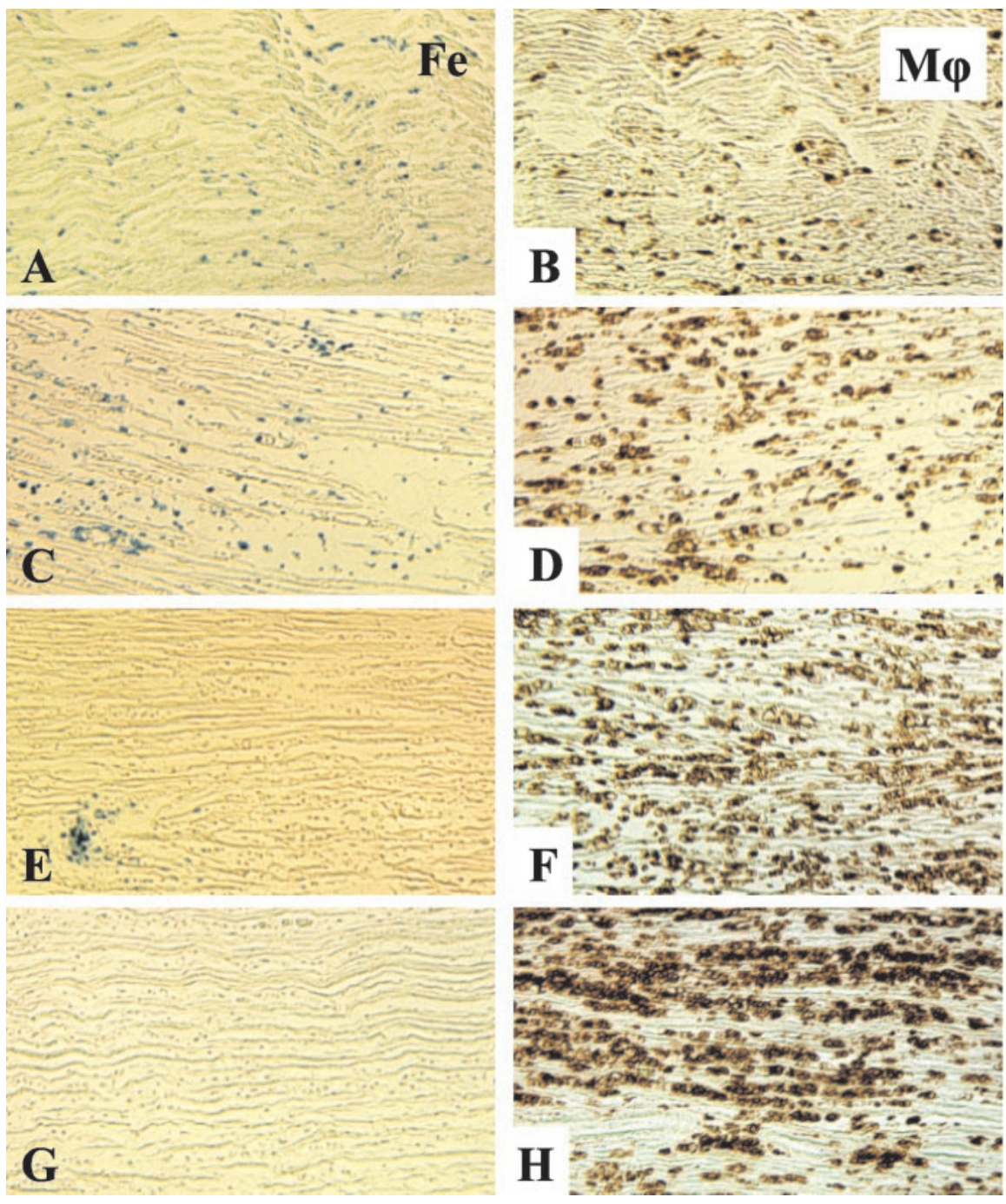

Figure 3. Time course of macrophage infiltration in crushed sciatic nerves: corresponding sections of distal nerve segments undergoing Wallerian degeneration at days $3(A, B), 5(C, D), 8(E, F)$, and $14(G, H)$ stained for iron $(F e)$ with Perl's stain $(A, C, E, G)$ and immunocytochemistry $(M \varphi)$ with the macrophage marker ED1 $(B, D, F, H)$. ED1 is a lysosomal antigen; its expression in macrophages increases along with their phagocytic activity. Note the increasing density of ED1 labeling from days 3 to $14(B-H)$. In contrast, iron labeling is restricted to early time points. At days 3 and $5(A, C)$, numerous blue iron-labeled macrophages are present. At day 8 , only small clusters of iron-positive cells are detectable $(E)$, whereas at day 14 virtually no iron deposits are found in the endoneurium $(G)$, despite the persistence of large numbers of ED1 + macrophages $(H)$. These findings correspond to the transient signal loss on MRI as shown in Figure 1. Magnifications (before 33\% reduction): $\mathrm{A}-\mathrm{H}, 140 \times$.

loss but did exhibit the typical unspecific signal increase on $\mathrm{T} 2-\mathrm{w}$ images indicating injury as described during WD (Titelbaum et al., 1989; Grant et al., 2002) (Fig. 1E).

To investigate whether SPIO application can differentiate between macrophage and microglial responses, we crushed ONs retro-orbitally and studied the distribution of iron particles in the degenerating intracranial proportions of the nerves at days 1, 2, 4, 7, 14, 21, and 28. Again, SPIOs were injected $24 \mathrm{hr}$ before histological analysis on paraffin sections. Virtually no iron deposition was seen in ON sections (data not shown), despite increasing levels of phagocytic activation revealed by ED1 immunoreactivity as described previously (Stoll et al. 1989b). Accordingly, no signal loss was detectable in ON scanned by MRI at days 4 and 14 after ON crush (Fig. $1 F$ ).

\section{Discussion}

As a principal finding, we show that macrophage infiltration can be visualized and tracked in vivo in an acute experimental nerve lesion. Macrophages entered crushed peripheral nerves with a proximodistal gradient starting at the lesion site, and their recruitment was restricted to a tight time frame of several days after injury. After nerve infiltration, macrophages remained stationary. In vivo mapping of macrophage infiltration was possible by injecting SPIO particles intracardially $24 \mathrm{hr}$ before MRI. SPIO particles are eliminated from the plasma by uptake of the mononuclear phagocyte system (Hamm et al., 1994). Local accumulation of SPIO particles leads to a signal loss on MR images because of their paramagnetic effect (Chen et al., 1999). In our study, areas of macrophage invasion could be identified as focal signal loss on MR images of crushed sciatic nerves that corresponded to the presence of iron-loaded ED1+ cells in tissue sections. Iron labeling in nerves was preferentially associated with small monocytes rather than large phagocytic macrophages, supporting the notion that these cells have been recruited only recently from the circulation.

The time course of macrophage entry into peripheral nerves in our study corresponds very well to the expression of the chemokine monocyte chemoattractant protein-1 (MCP-1). MCP-1 was upregulated within $3 \mathrm{hr}$ after nerve crush in Schwann cells at the lesion site and in more distal segments from $24 \mathrm{hr}$ to day 5 (Toews et al., 1998; Subang and Richardson, 2001; Tofaris et al., 2002). In support of a critical role of MCP-1 in macrophage recruitment, transgenic mice lacking cysteinecysteine chemokine receptor 2 , the main macrophage receptor for MCP-1, showed impaired macrophage invasion after nerve injury and a significant decrease in myelin clearance (Siebert et al., 2000). The abrupt disappearance of iron-mediated signal loss in crushed nerve after day 8 corresponds to downregulation of MCP- 1 . The fact that endoneurial macrophages at later stages of WD did not phagocytose systemically injected SPIO particles argues against passive diffusion of SPIO into nerves through the leaky blood-nerve barrier and subsequent local SPIO particle uptake. Disturbance of the blood-brain barrier in WD by far outlasts the signal loss observed on MR images (Seitz et al., 1989).

Our notion that macrophage recruitment is a transient event was further confirmed by a sequential crush experiment that revealed signal loss caused by iron-labeled macrophages only within nerves that had been crushed $5 \mathrm{~d}$ before. By contrast, nerves that had been crushed $11 \mathrm{~d}$ before in the same animals revealed a hyperintense signal as an unspecific sign for nerve damage but no areas of signal loss indicative of infiltration by iron-labeled macrophages. A persistent prolongation of the T2 relaxation time as seen in the 11-d-old crushed nerves is a typical finding in experimental and human nerve lesions that normalizes only after successful nerve regeneration (Titelbaum et al., 1989; 
Dailey et al., 1997). The prolongation of the T2 relaxation time has been attributed to various, nonspecific processes such as demyelination, inflammation, and axonal loss (Stanisz et al., 2001). In contrast, signal loss on SPIO-enhanced MRI specifically identifies areas of recent macrophage entry.

It is of note that no SPIO particle accumulation was seen in the distal segments of optic nerves undergoing WD. We thereby support the notion that hematogenous macrophages do not infiltrate degenerating central nerves (optic nerve) or fiber tracts remote from the lesion site as indicated by previous immunocytochemical studies (Perry et al., 1987; George and Griffin, 1994). Thus, SPIO labeling can differentiate between microglial and acute hematogenous macrophage responses in the nervous system. This opens a broad range of possible experimental and clinical applications. It is possible to determine in vivo the dynamics of macrophage infiltration that is instrumental in autoimmune and infectious disorders, stroke, and possibly also neurodegenerative disorders (Benveniste, 1997; Stoll and Jander, 1999; Kiefer et al., 2001; Williams and Hickey, 2002) and to monitor antiinflammatory treatment strategies. Because commercially available SPIO particles (Resovist) that are commonly applied to diagnose liver tumors (Shamsi et al., 1998) were used in our study, our technique of macrophage mapping may be applicable to human use.

\section{References}

Banati RB (2002) Visualising microglial activation in vivo. Glia 40:206-217. Benveniste EN (1997) Role of macrophages/microglia in multiple sclerosis and experimental allergic encephalomyelitis. J Mol Med 75:165-173.

Brück W (1997) The role of macrophages in Wallerian degeneration. Brain Pathol 7:741-752.

Casselman JW, Kuhweide R, Deimling M, Ampe W, Dehaene I, Meeus L (1993) Constructive interference in steady state-3DFT MR imaging of the inner ear and cerebellopontine angle. AJNR Am J Neuroradiol 14:47-57.

Chen F, Ward J, Robinson PJ (1999) MR imaging of the liver and spleen: a comparison of the effects on signal intensity of two superparamagnetic iron oxide agents. Magn Reson Imaging 17:549-556.

Dailey AT, Tsuruda JS, Filler AG, Maravilla KR, Goodkin R, Kliot M (1997) Magnetic resonance neurography of peripheral nerve degeneration and regeneration. Lancet 350:1221-1222.

David S, Ousman SS (2002) Recruiting the immune response to promote axon regeneration in the injured spinal cord. Neuroscientist 8:33-41.

Flaris NA, Densmore TL, Molleston MC, Hickey WF (1993) Characterization of microglia and macrophages in the central nervous system of rats: definition of the differential expression of molecules using standard and novel monoclonal antibodies in normal CNS and in four models of parenchymal reaction. Glia 7:34-40.

George R, Griffin JW (1994) Delayed macrophage responses and myelin clearance during Wallerian degeneration in the central nervous system: the dorsal radiculotomy model. Exp Neurol 129:225-236.

Grant GA, Britz GW, Goodkin R, Jarvik JG, Maravilla K, Kliot M (2002) The utility of magnetic resonance imaging in evaluation peripheral nerve disorders. Muscle Nerve 25:314-331.

Hamm B, Staks T, Taupitz M, Maibauer R, Speidel A, Huppertz A, Frenzel T, Lawaczeck R, Wolf KJ, Lange L (1994) Contrast-enhanced MR imaging of liver and spleen: first experience in humans with a new superparamagnetic iron oxide. J Magn Reson Imaging 4:659-668.

Higer HP, Bielke G (1990) Tissue characterization in MR-imaging. New York: Springer.
Horner PJ, Gage FH (2000) Regenerating the damaged central nervous system. Nature 407:963-970.

Kiefer R, Kieseier BC, Stoll G, Hartung HP (2001) The role of macrophages in immune-mediated damage to the peripheral nervous system. Prog Neurobiol 64:109-127.

Kuhlmann T, Bitsch A, Stadelmann C, Siebert H, Brück W (2001) Macrophages are eliminated from injured peripheral nerve via local apoptosis and circulation to regional lymph nodes and the spleen. J Neurosci 21:3401-3408.

Kuhn MJ, Johnson KA, Davis KR (1988) Wallerian degeneration: evaluation with MR imaging. Radiology 168:199-202.

Perry VH, Brown MC, Gordon S (1987) The macrophage response to central and peripheral nerve injury. A possible role for macrophages in regeneration. J Exp Med 165:1218-1223.

Ramon y Cajal S (1991) Cajal's degeneration and regeneration of the nervous system (May RM, DeFelipe J, and Jones EG, eds). Oxford: Oxford UP.

Seitz RJ, Reiners K, Himmelmann F, Heininger K, Hartung HP, Toyka KV (1989) The blood-nerve barrier in Wallerian degeneration: a sequential long-term study. Muscle Nerve 12:627-635.

Shamsi K, Balzer T, Saini S, Ros PR, Nelson RC, Carter EC, Tollerfield S, Niendorf HP (1998) Superparamagnetic iron oxide particles (SH U 555 A): evaluation of efficacy in three doses for hepatic MR imaging. Radiology 206:365-371.

Siebert H, Sachse A, Kuziel WA, Maeda N, Brück W (2000) The chemokine receptor CCR2 is involved in macrophage recruitment to the injured peripheral nervous system. J Neuroimmunol 110:177-185.

Snider WD, Zhou FQ, Zhong J, Markus A (2002) Signaling the pathway to regeneration. Neuron 35:13-16.

Stanisz GJ, Midha R, Munro CA, Henkelman RM (2001) MR properties of rat sciatic nerve following trauma. Magn Reson Med 45:415-420.

Stoll G, Jander S (1999) The role of microglia and macrophages in the pathophysiology of the CNS. Prog Neurobiol 58:233-247.

Stoll G, Müller HW (1999) Nerve injury, axonal degeneration and neural regeneration: basic insights. Brain Pathol 9:313-325.

Stoll G, Griffin JW, Li CY, Trapp BD (1989a) Wallerian degeneration of the peripheral nervous system: participation of both Schwann cells and macrophages in myelin degradation. J Neurocytol 18:671-683.

Stoll G, Trapp BD, Griffin JW (1989b) Macrophage function during Wallerian degeneration of the rat optic nerve: clearance of degenerating myelin and Ia expression. J Neurosci 9:2327-2335.

Subang MC, Richardson PM (2001) Influence of injury and cytokines on synthesis of monocyte chemoattractant protein-1 mRNA in peripheral nerve tissue. Eur J Neurosci 13:521-528.

Titelbaum DS, Frazier JL, Grossman RI, Joseph PM, Yu LT, Kassab EA, Hickey WF, LaRossa D, Brown MJ (1989) Wallerian degeneration and inflammation in rat peripheral nerve detected by in vivo MR imaging. AJNR Am J Neuroradiol 10:741-746.

Toews AD, Barret C, Morell P (1998) Monocyte chemoattractant protein 1 is responsible for macrophage recruitment following injury to sciatic nerve. J Neurosci Res 53:260-267.

Tofaris GK, Patterson PH, Jessen KR, Mirsky R (2002) Denervated Schwann cells attract macrophages by secretion of leukemia inhibitory factor (LIF) and macrophage attractant protein-1 (MCP-1) in a process regulated by interleukin-6 and LIF. J Neurosci 22:6696-6703.

Waller A (1850) Experiments on the section of the glossopharyngeal and hypoglossal nerves of the frog, and observations of the alterations produced thereby in the structure of their primitive fibres. Philos Trans R Soc Lond B Biol Sci 140:423-429.

Williams KC, Hickey WF (2002) CNS damage, monocytes and macrophages, and neurological disorders in AIDS. Annu Rev Neurosci 25:537562 . 